\title{
Common fuzzy fixed points for fuzzy mappings
}

\section{Akbar Azam ${ }^{1}$ and Ismat Beg²*}

"Correspondence:

begismat@yahoo.com

${ }^{2}$ Centre for Applicable Mathematics and Statistics, University of Central

Punjab, Lahore, 54770, Pakistan

Full list of author information is

available at the end of the article

\begin{abstract}
Let $(X, d)$ be a metric space and $S, T$ be mappings from $X$ to a set of all fuzzy subsets of $X$. We obtained sufficient conditions for the existence of a common $\alpha$-fuzzy fixed point of $S$ and $T$.
\end{abstract}

Keywords: analysis; fuzzy set; fuzzy mapping; $\alpha$-fuzzy fixed point

\section{Introduction}

Fixed point theorems play a fundamental role in demonstrating the existence of solutions to a wide variety of problems arising in mathematics, physics, engineering, medicine and social sciences. The study of fixed point theorems in fuzzy mathematics was instigated by Weiss [1], Butnariu [2], Singh and Talwar [3], Estruch and Vidal [4], Wang et al. [5], Mihet [6], Qiu et al. [7] and Beg and Abbas [8]. Heilpern [9] introduced the concept of fuzzy contraction mappings and established the fuzzy Banach contraction principle on a complete metric linear spaces with the $d_{\infty}$-metric for fuzzy sets. Azam and Beg [10] proved common fixed point theorems for a pair of fuzzy mappings satisfying Edelstein, Alber and Guerr-Delabriere type contractive conditions in a metric linear space. Azam et al. [11] presented some fixed point theorems for fuzzy mappings under Edelstein locally contractive conditions on a compact metric space with the $d_{\infty}$-metric for fuzzy sets. Frigon and Regan [12] generalized the Heilpern theorem under a contractive condition for 1-level sets (i.e., $\left.[T x]_{1}\right)$ of a fuzzy contraction $T$ on a complete metric space, where 1-level sets are not assumed to be convex and compact. Amemiya and Takahashi [13] studied some mathematical properties of contractive type set-valued and fuzzy mappings to obtain fixed points of fuzzy mappings by using the concept of $w$-distance (see [13]) in complete metric spaces. Recently, Zhang et al. [14] proved some common fixed point theorems for contraction mappings in a new fuzzy metric space.

The aim of this paper is to obtain a common $\alpha$-fuzzy fixed point of a pair of fuzzy mappings $S$ and $T$ on a complete metric space under a generalized contractive condition for $\alpha$-level sets (i.e., $[S x]_{\alpha},[T x]_{\alpha}$ ) of $S$ and $T$ in connection with Hausdorff metric for fuzzy sets. Our result (Theorem 5) generalizes the results proved by Azam and Arshad [15, Theorem 4], Bose and Sahani [16] and Vijayaraju and Marudai [17, Theorem 3.1] among others. 


\section{Preliminaries}

Let $(X, d)$ be a metric space and $C B(X)$ be the family of nonempty closed and bounded subsets of $X$. For $A, B \in C B(X)$, define

$$
H(A, B)=\max \left\{\sup _{a \in A} d(a, B), \sup _{b \in B} d(A, b)\right\}
$$

where

$$
d(x, A)=\inf _{y \in A} d(x, y)
$$

A fuzzy set in $X$ is a function with domain $X$ and values in $[0,1]$. If $A$ is a fuzzy set and $x \in X$, then the function-value $A(x)$ is called the grade of membership of $x$ in $A$. The $\alpha$-level set of $A$ is denoted by $[A]_{\alpha}$ and is defined as follows:

$$
\begin{aligned}
& {[A]_{\alpha}=\{x: A(x) \geq \alpha\} \quad \text { if } \alpha \in(0,1],} \\
& {[A]_{0}=\overline{\{x: A(x)>0\}} .}
\end{aligned}
$$

Here, $\bar{B}$ denotes the closure of the set $B$. Let $\mathcal{F}(X)$ be the collection of all fuzzy sets in a metric space $X$. For $A, B \in \mathcal{F}(X), A \subset B$ means $A(x) \leq B(x)$ for each $x \in X$. We denote the fuzzy set $\chi_{\{x\}}$ by $\{x\}$ unless and until it is stated, where $\chi_{A}$ is the characteristic function of the crisp set $A$. If there exists an $\alpha \in[0,1]$ such that $[A]_{\alpha},[B]_{\alpha} \in C B(X)$, then define

$$
\begin{aligned}
& p_{\alpha}(A, B)=\inf _{x \in[A]_{\alpha}, y \in[B]_{\alpha}} d(x, y), \\
& D_{\alpha}(A, B)=H\left([A]_{\alpha},[B]_{\alpha}\right) .
\end{aligned}
$$

If $[A]_{\alpha},[B]_{\alpha} \in C B(X)$ for each $\alpha \in[0,1]$, then define

$$
\begin{aligned}
& p(A, B)=\sup _{\alpha} p_{\alpha}(A, B), \\
& d_{\infty}(A, B)=\sup _{\alpha} D_{\alpha}(A, B) .
\end{aligned}
$$

We write $p(x, B)$ instead of $p(\{x\}, B)$. A fuzzy set $A$ in a metric linear space $V$ is said to be an approximate quantity if and only if $[A]_{\alpha}$ is compact and convex in $V$ for each $\alpha \in[0,1]$ and

$$
\sup _{x \in V} A(x)=1
$$

We denote the collection of all approximate quantities in a metric linear space $X$ by $W(X)$. Let $X$ be an arbitrary set, $Y$ be a metric space. A mapping $T$ is called a fuzzy mapping if $T$ is a mapping from $X$ into $\mathcal{F}(Y)$. A fuzzy mapping $T$ is a fuzzy subset on $X \times Y$ with a membership function $T(x)(y)$. The function $T(x)(y)$ is the grade of membership of $y$ in $T(x)$.

Definition 1 Let $S, T$ be fuzzy mappings from $X$ into $\mathcal{F}(X)$. A point $z$ in $X$ is called an $\alpha$-fuzzy fixed point of $T$ if $z \in[T z]_{\alpha}$. The point $z$ is called a common $\alpha$-fuzzy fixed point of $S$ and $T$ if $z \in[S z]_{\alpha} \cap[T z]_{\alpha}$. When $\alpha=1$, it is called a common fixed point of fuzzy maps. 
For the sake of convenience, we first state some known results for subsequent use in the next section.

Lemma $2[18]$ Let $(X, d)$ be a metric space and $A, B \in C B(X)$, then for each $a \in A$,

$$
d(a, B) \leq H(A, B)
$$

Lemma 3 [18] Let $(X, d)$ be a metric space and $A, B \in C B(X)$, then for each $a \in A, \beta>0$, there exists an element $b \in B$ such that $d(a, b) \leq H(A, B)+\beta$.

Lemma 4 [19] Let $V$ be a metric linear space, $T: V \rightarrow W(V)$ be a fuzzy mapping and $x_{0} \in V$. Then there exists $x_{1} \in V$ such that $\left\{x_{1}\right\} \subset T\left(x_{0}\right)$.

\section{Common fuzzy fixed points}

In this section, we establish Theorem 5 on the existence of an $\alpha$-fuzzy fixed point of a fuzzy mapping and also obtain a fixed point of fuzzy mappings (see Corollaries 8 and 9) and multivalued mappings (see Corollary 7).

Theorem 5 Let $(X, d)$ be a complete metric space and let $S, T$ be fuzzy mappings from $X$ to $\mathcal{F}(X)$ satisfying the following conditions:

(a) for each $x \in X$, there exists $\alpha(x) \in(0,1]$ such that $[S x]_{\alpha(x)},[T x]_{\alpha(x)}$ are nonempty closed bounded subsets of $X$ and

(b)

$$
\begin{aligned}
H\left([S x]_{\alpha(x)},[T y]_{\alpha(y)}\right) \leq & a_{1} d\left(x,[S x]_{\alpha(x)}\right)+a_{2} d\left(y,[T y]_{\alpha(y)}\right) \\
& +a_{3} d\left(x,[T y]_{\alpha(y)}\right)+a_{4} d\left(y,[S x]_{\alpha(x)}\right)+a_{5} d(x, y)
\end{aligned}
$$

for all $x, y \in X$, where $a_{1}, a_{2}, a_{3}, a_{4}, a_{5}$ are nonnegative real numbers and $\sum_{i=1}^{5} a_{i}<1$ and either $a_{1}=a_{2}$ or $a_{3}=a_{4}$. Then there exists $z \in X$ such that $z \in[S z]_{\alpha(z)} \cap[T z]_{\alpha(z)}$.

Proof We consider the following three possible cases:

(i) $a_{1}+a_{3}+a_{5}=0$;

(ii) $a_{2}+a_{4}+a_{5}=0$;

(iii) $a_{1}+a_{3}+a_{5} \neq 0, a_{2}+a_{4}+a_{5} \neq 0$.

Case (i): For $x \in X$, there exists $\alpha(x) \in(0,1]$ such that $[S x]_{\alpha(x)}$ is a nonempty closed bounded subset of $X$. Take $y \in[S x]_{\alpha(x)}$ and similarly $z \in[T y]_{\alpha(y)}$. Then by Lemma 2, we obtain

$$
d\left(y,[T y]_{\alpha(y)}\right) \leq H\left([S x]_{\alpha(x)},[T y]_{\alpha(y)}\right) .
$$

Now, inequality (1) implies that

$$
\begin{aligned}
d\left(y,[T y]_{\alpha(y)}\right) \leq & a_{1} d\left(x,[S x]_{\alpha(x)}\right)+a_{2} d\left(y,[T y]_{\alpha(y)}\right)+a_{3} d\left(x,[T y]_{\alpha(y)}\right) \\
& +a_{4} d\left(y,[S x]_{\alpha(x)}\right)+a_{5} d(x, y) .
\end{aligned}
$$

Using $a_{1}+a_{3}+a_{5}=0$ together with the fact that $d\left(y,[S x]_{\alpha(x)}\right)=0$, we obtain

$$
d\left(y,[T y]_{\alpha(y)}\right) \leq a_{2} d\left(y,[T y]_{\alpha(y)}\right)
$$


It follows that $y \in[T y]_{\alpha(y)}$, which further implies that

$$
d\left(y,[S y]_{\alpha(y)}\right) \leq H\left([T y]_{\alpha(y)},[S y]_{\alpha(y)}\right) .
$$

Again, inequality (1) gives $d\left(y,[S y]_{\alpha(y)}\right)=0$. It follows that

$$
y \in[S y]_{\alpha(y)} \cap[T y]_{\alpha(y)} .
$$

Case (ii): It can be easily seen that

$$
z \in[S z]_{\alpha(z)} \cap[T z]_{\alpha(z)} .
$$

Case (iii): Let

$$
\lambda=\left(\frac{a_{1}+a_{3}+a_{5}}{1-a_{2}-a_{3}}\right), \quad \mu=\left(\frac{a_{2}+a_{4}+a_{5}}{1-a_{1}-a_{4}}\right) .
$$

If $a_{3}=a_{4}$, then $\lambda, \mu<1$ and so $0<\lambda \mu<1$. Moreover, if $a_{1}=a_{2}$,

$$
\begin{aligned}
0 & <\lambda \mu=\left(\frac{a_{1}+a_{3}+a_{5}}{1-a_{2}-a_{3}}\right)\left(\frac{a_{2}+a_{4}+a_{5}}{1-a_{1}-a_{4}}\right) \\
& =\left(\frac{a_{1}+a_{3}+a_{5}}{1-a_{1}-a_{3}}\right)\left(\frac{a_{1}+a_{4}+a_{5}}{1-a_{1}-a_{4}}\right) \\
& =\left(\frac{a_{1}+a_{3}+a_{5}}{1-a_{1}-a_{4}}\right)\left(\frac{a_{1}+a_{4}+a_{5}}{1-a_{1}-a_{3}}\right)<1 .
\end{aligned}
$$

Choose $x_{0} \in X$, then by hypotheses there exists $\alpha\left(x_{0}\right) \in(0,1]$ such that $\left[S x_{0}\right]_{\alpha\left(x_{0}\right)}$ is a nonempty closed bounded subset of $X$. For convenience, we denote $\alpha\left(x_{0}\right)$ by $\alpha_{1}$. Let $x_{1} \in\left[S x_{0}\right]_{\alpha_{1}}$; for this $x_{1}$, there exists $\alpha_{2} \in(0,1]$ such that $\left[T x_{1}\right]_{\alpha_{2}}$ is a nonempty closed bounded subset of $X$. Since $a_{1}+a_{3}+a_{5}>0$, by Lemma 3, there exists $x_{2} \in\left[T x_{1}\right]_{\alpha_{2}}$ such that

$$
d\left(x_{1}, x_{2}\right) \leq H\left(\left[S x_{0}\right]_{\alpha_{1}},\left[T x_{1}\right]_{\alpha_{2}}\right)+a_{1}+a_{3}+a_{5} .
$$

It implies that

$$
\begin{aligned}
d\left(x_{1}, x_{2}\right) \leq & H\left(\left[S x_{0}\right]_{\alpha_{1}},\left[T x_{1}\right]_{\alpha_{2}}\right)+a_{1}+a_{3}+a_{5} \\
\leq & a_{1} d\left(x_{0},\left[S x_{0}\right]_{\alpha_{1}}\right)+a_{2} d\left(x_{1},\left[T x_{1}\right]_{\alpha_{2}}\right)+a_{3} d\left(x_{0},\left[T x_{1}\right]_{\alpha_{2}}\right) \\
& +a_{4} d\left(x_{1},\left[S x_{0}\right]_{\alpha_{1}}\right)+a_{5} d\left(x_{0}, x_{1}\right)+a_{1}+a_{3}+a_{5} \\
\leq & \left(a_{1}+a_{5}\right) d\left(x_{0}, x_{1}\right)+a_{2} d\left(x_{1}, x_{2}\right)+a_{3} d\left(x_{0}, x_{2}\right)+a_{1}+a_{3}+a_{5} \\
\leq & \left(a_{1}+a_{3}+a_{5}\right) d\left(x_{0}, x_{1}\right)+\left(a_{2}+a_{3}\right) d\left(x_{1}, x_{2}\right)+a_{1}+a_{3}+a_{5} \\
\leq & \left(a_{1}+a_{3}+a_{5}\right) d\left(x_{0}, x_{1}\right)+a_{1}+a_{3}+a_{5} \\
\leq & \lambda d\left(x_{0}, x_{1}\right)+\lambda .
\end{aligned}
$$


By the same argument, we can find $\alpha_{3} \in(0,1]$ and $x_{3} \in\left[S x_{2}\right]_{\alpha_{3}}$ such that

$$
\begin{aligned}
d\left(x_{2}, x_{3}\right) \leq & H\left(\left[S x_{2}\right]_{\alpha_{3}},\left[T x_{1}\right]_{\alpha_{2}}\right)+\lambda\left(a_{2}+a_{4}+a_{5}\right) \\
\leq & a_{1} d\left(x_{2},\left[S x_{2}\right]_{\alpha_{3}}\right)+a_{2} d\left(x_{1},\left[T x_{1}\right]_{\alpha_{2}}\right)+a_{3} d\left(x_{2},\left[T x_{1}\right]_{\alpha_{2}}\right) \\
& +a_{4} d\left(x_{1},\left[S x_{2}\right]_{\alpha_{3}}\right)+a_{5} d\left(x_{2}, x_{1}\right)+\lambda\left(a_{2}+a_{4}+a_{5}\right) \\
\leq & a_{1} d\left(x_{2}, x_{3}\right)+a_{2} d\left(x_{1}, x_{2}\right)+a_{3} d\left(x_{2}, x_{2}\right) \\
& +a_{4} d\left(x_{1}, x_{3}\right)+a_{5} d\left(x_{2}, x_{1}\right)+\lambda\left(a_{2}+a_{4}+a_{5}\right) \\
\leq & \left(a_{2}+a_{4}+a_{5}\right) d\left(x_{1}, x_{2}\right)+\left(a_{1}+a_{4}\right) d\left(x_{2}, x_{3}\right)+\lambda\left(a_{2}+a_{4}+a_{5}\right) \\
\leq & \mu d\left(x_{1}, x_{2}\right)+\lambda \mu
\end{aligned}
$$

By induction, we produce a sequence $\left\{x_{n}\right\}$ of points of $X$,

$$
\begin{aligned}
& x_{2 k+1} \in\left[S x_{2 k}\right]_{\alpha_{2 k+1}}, \\
& x_{2 k+2} \in\left[T x_{2 k+1}\right]_{\alpha_{2 k+2}}, \quad k=0,1,2, \ldots,
\end{aligned}
$$

such that

$$
\begin{aligned}
& d\left(x_{2 k+1}, x_{2 k+2}\right) \leq H\left(\left[S x_{2 k}\right]_{\alpha_{2 k+1}},\left[T x_{2 k+1}\right]_{\alpha_{2 k+2}}\right)+(\lambda \mu)^{k}\left(a_{1}+a_{3}+a_{5}\right), \\
& d\left(x_{2 k+2}, x_{2 k+3}\right) \leq H\left(\left[S x_{2 k+2}\right]_{\alpha_{2 k+3}},\left[T x_{2 k+1}\right]_{\alpha_{2 k+2}}\right)+(\lambda \mu)^{k} \lambda\left(a_{2}+a_{4}+a_{5}\right) .
\end{aligned}
$$

It implies that

$$
\begin{aligned}
d\left(x_{2 k+1}, x_{2 k+2}\right) \leq & a_{1} d\left(x_{2 k},\left[S x_{2 k}\right]_{\alpha_{2 k+1}}\right)+a_{2} d\left(x_{2 k+1},\left[T x_{2 k+1}\right]_{\alpha_{2 k+2}}\right) \\
& +a_{3} d\left(x_{2 k},\left[T x_{2 k+1}\right]_{\alpha_{2 k+2}}\right)+a_{4} d\left(x_{2 k+1},\left[S x_{2 k}\right]_{\alpha_{2 k+1}}\right) \\
& +a_{5} d\left(x_{2 k}, x_{2 k+1}\right)+(\lambda \mu)^{k}\left(a_{1}+a_{3}+a_{5}\right) \\
\leq & \left(a_{1}+a_{3}+a_{5}\right) d\left(x_{2 k}, x_{2 k+1}\right)+\left(a_{2}+a_{3}\right) d\left(x_{2 k+1}, x_{2 k+2}\right) \\
& +(\lambda \mu)^{k}\left(a_{1}+a_{3}+a_{5}\right) \\
\leq & \lambda d\left(x_{2 k}, x_{2 k+1}\right)+\lambda(\lambda \mu)^{k} .
\end{aligned}
$$

Similarly,

$$
\begin{aligned}
d\left(x_{2 k+2}, x_{2 k+3}\right) \leq & a_{1} d\left(x_{2 k+2},\left[S x_{2 k+2}\right]_{\alpha_{2 k+3}}\right)+a_{2} d\left(x_{2 k+1},\left[T x_{2 k+1}\right]_{\alpha_{2 k+2}}\right) \\
& +a_{3} d\left(x_{2 k+2},\left[T x_{2 k+1}\right]_{\alpha_{2 k+2}}\right)+a_{4} d\left(x_{2 k+1},\left[S x_{2 k+2}\right]_{\alpha_{2 k+3}}\right) \\
& +a_{5} d\left(x_{2 k+2}, x_{2 k+1}\right)+(\lambda \mu)^{k} \lambda\left(a_{2}+a_{4}+a_{5}\right) \\
\leq & \left(a_{2}+a_{4}+a_{5}\right) d\left(x_{2 k+1}, x_{2 k+2}\right)+\left(a_{1}+a_{4}\right) d\left(x_{2 k+2}, x_{2 k+3}\right) \\
& +(\lambda \mu)^{k} \lambda\left(a_{2}+a_{4}+a_{5}\right) \\
\leq & \mu d\left(x_{2 k+1}, x_{2 k+2}\right)+(\lambda \mu)^{k+1}
\end{aligned}
$$


It follows that for each $k=0,1,2, \ldots$,

$$
\begin{aligned}
d\left(x_{2 k+1}, x_{2 k+2}\right) & \leq \lambda d\left(x_{2 k}, x_{2 k+1}\right)+\lambda(\lambda \mu)^{k} \\
& \leq \lambda\left[\mu d\left(x_{2 k-1}, x_{2 k}\right)+(\lambda \mu)^{k}\right]+\mu^{k} \lambda^{k+1} \\
& \leq \lambda \mu d\left(x_{2 k-1}, x_{2 k}\right)+2 \lambda(\lambda \mu)^{k} \\
& \leq \lambda \mu \lambda d\left(x_{2 k-2}, x_{2 k-1}\right)+3 \lambda(\lambda \mu)^{k} \\
& \leq \cdots \leq \lambda(\lambda \mu)^{k} d\left(x_{0}, x_{1}\right)+(2 k+1) \lambda(\lambda \mu)^{k}
\end{aligned}
$$

and

$$
\begin{aligned}
d\left(x_{2 k+2}, x_{2 k+3}\right) & \leq \mu d\left(x_{2 k+1}, x_{2 k+2}\right)+(\lambda \mu)^{k+1} \\
& \leq \cdots \leq(\lambda \mu)^{k+1} d\left(x_{0}, x_{1}\right)+(2 k+2)(\lambda \mu)^{k+1} .
\end{aligned}
$$

Then for $p<q$, we have

$$
\begin{aligned}
d\left(x_{2 p+1}, x_{2 q+1}\right) \leq & d\left(x_{2 p+1}, x_{2 p+2}\right)+d\left(x_{2 p+2}, x_{2 p+3}\right)+d\left(x_{2 p+3}, x_{2 p+4}\right) \\
& +\cdots+d\left(x_{2 q}, x_{2 q+1}\right) \\
\leq & {\left[\lambda \sum_{i=p}^{q-1}(\lambda \mu)^{i}+\sum_{i=p+1}^{q}(\lambda \mu)^{i}\right] d\left(x_{0}, x_{1}\right) } \\
& +\lambda \sum_{i=p}^{q-1}(2 i+1)(\lambda \mu)^{i}+\sum_{i=p+1}^{q} 2 i(\lambda \mu)^{i} .
\end{aligned}
$$

Similarly, we obtain

$$
\begin{aligned}
d\left(x_{2 p}, x_{2 q+1}\right) \leq & {\left[\sum_{i=p}^{q}(\lambda \mu)^{i}+\lambda \sum_{i=p}^{q-1}(\lambda \mu)^{i}\right] d\left(x_{0}, x_{1}\right) } \\
& +\sum_{i=p}^{q} 2 i(\lambda \mu)^{i}+\lambda \sum_{i=p}^{q-1}(2 i+1)(\lambda \mu)^{i}, \\
d\left(x_{2 p}, x_{2 q}\right) \leq & {\left[\sum_{i=p}^{q-1}(\lambda \mu)^{i}+\lambda \sum_{i=p}^{q-1}(\lambda \mu)^{i}\right] d\left(x_{0}, x_{1}\right) } \\
& +\sum_{i=p}^{q-1} 2 i(\lambda \mu)^{i}+\lambda \sum_{i=p}^{q-1}(2 i+1)(\lambda \mu)^{i}
\end{aligned}
$$

and

$$
\begin{aligned}
d\left(x_{2 p+1}, x_{2 q}\right) \leq & {\left[\lambda \sum_{i=p}^{q-1}(\lambda \mu)^{i}+\sum_{i=p+1}^{q-1}(\lambda \mu)^{i}\right] d\left(x_{0}, x_{1}\right) } \\
& +\sum_{i=p}^{q-1}(2 i+1)(\lambda \mu)^{i}+\sum_{i=p+1}^{q-1} 2 i(\lambda \mu)^{i} .
\end{aligned}
$$


Since $(\lambda \mu)<1$, it follows from Cauchy's root test $\sum 2 i(\lambda \mu)^{i}, \sum(2 i+1)(\lambda \mu)^{i}$ are convergent series. Therefore, $\left\{x_{n}\right\}$ is a Cauchy sequence. Since $X$ is complete, there exists $u \in X$ such that $x_{n} \rightarrow u$. Now,

$$
\begin{aligned}
d\left(u,[S u]_{\alpha(u)}\right) \leq & d\left(u, x_{2 n}\right)+d\left(x_{2 n},[S u]_{\alpha(u)}\right) \\
\leq & d\left(u, x_{2 n}\right)+H\left(\left[T x_{2 n-1}\right]_{2 n},[S u]_{\alpha(u)}\right) \\
\leq & d\left(u, x_{2 n}\right)+a_{1} d\left(u,[S u]_{\alpha(u)}\right)+a_{2} d\left(x_{2 n-1},\left[T x_{2 n-1}\right] \alpha_{2 n}\right) \\
& +a_{3} d\left(u,\left[T x_{2 n-1}\right] \alpha_{2 n}\right)+a_{4} d\left(x_{2 n-1},[S u]_{\alpha(u)}\right)+a_{5} d\left(u, x_{2 n-1}\right) \\
\leq & d\left(u, x_{2 n}\right)+a_{1} d\left(u,[S u]_{\alpha(u)}\right)+a_{2} d\left(x_{2 n-1}, x_{2 n}\right) \\
& +a_{3} d\left(u, x_{2 n}\right)+a_{4}\left[d\left(x_{2 n-1}, u\right)+d\left(u,[S u]_{\alpha(u)}\right)\right]+a_{5} d\left(u, x_{2 n-1}\right) \\
\leq & \left(1+a_{3}\right) d\left(u, x_{2 n}\right)+\left(a_{5}+a_{4}\right) d\left(u, x_{2 n-1}\right)+a_{2} d\left(x_{2 n-1}, x_{2 n}\right) \\
& +\left(a_{1}+a_{4}\right) d\left(u,[S u]_{\alpha(u)}\right) .
\end{aligned}
$$

It further implies that

$$
\begin{aligned}
d\left(u,[S u]_{\alpha(u)}\right) \leq & {\left[\frac{1+a_{3}}{1-a_{1}-a_{4}}\right] d\left(u, x_{2 n}\right)+\left[\frac{a_{4}+a_{5}}{1-a_{1}-a_{4}}\right] d\left(u, x_{2 n-1}\right) } \\
& +\left[\frac{a_{2}}{1-a_{1}-a_{4}}\right] d\left(x_{2 n-1}, x_{2 n}\right) .
\end{aligned}
$$

Letting $n \rightarrow \infty$, we have $d\left(u,[S u]_{\alpha(u)}\right)=0$. It implies that $u \in[S u]_{\alpha(u)}$. Similarly, by using

$$
d\left(u,[T u]_{\alpha(u)}\right) \leq d\left(u, x_{2 n+1}\right)+d\left(x_{2 n+1},[T u]_{\alpha(u)}\right)
$$

we can show that $u \in[T u]_{\alpha(u)}$, which implies that $u \in[S u]_{\alpha(u)} \cap[\mathrm{Tu}]_{\alpha(u)}$.

The proof of the following corollary illustrates a link between multivalued mappings and fuzzy mappings. It is well known [20] that

$$
(X, d) \mapsto(C B(X), H) \mapsto\left(E(X), d_{\infty}\right)
$$

are isometric embeddings under $x \rightarrow\{x\}$ and $A \rightarrow \chi_{A}$, respectively, where

$$
E(X)=\left\{A \in F(X):[A]_{\alpha} \in C B(X), \forall \alpha \in[0,1]\right\}
$$

and $\{x\}$ is a crisp set.

In the following, we furnish an illustrative example to highlight the utility of Theorem 5.

Example 6 Let $X=\{1,2,3\},\{1\},\{2\},\{3\}$ be crisp sets. Define $d: X \times X \rightarrow \mathbb{R}$ as follows:

$$
d(x, y)= \begin{cases}0 & \text { if } x=y, \\ \frac{5}{17} & \text { if } x \neq y \text { and } x, y \in X-\{2\}, \\ 1 & \text { if } x \neq y \text { and } x, y \in X-\{3\}, \\ \frac{4}{17} & \text { if } x \neq y \text { and } x, y \in X-\{1\} .\end{cases}
$$


Define fuzzy mappings $S, T: X \rightarrow \mathcal{F}(X)$ as follows:

$$
\begin{gathered}
T(1)(t)=T(3)(t)= \begin{cases}\frac{3}{4} & \text { if } t=1, \\
\frac{1}{2} & \text { if } t=2, \\
0 & \text { if } t=3,\end{cases} \\
T(2)(t)= \begin{cases}0 & \text { if } t=1, \\
\frac{1}{3} & \text { if } t=2, \\
\frac{3}{4} & \text { if } t=3,\end{cases}
\end{gathered}
$$

and

$$
S(1)(t)=S(2)(t)=S(3)(t)= \begin{cases}\frac{3}{4} & \text { if } t=1 \\ \frac{1}{19} & \text { if } t=2 \\ 0 & \text { if } t=3\end{cases}
$$

Then, for $\alpha(x)=\frac{3}{4}$,

$$
[T x]_{\alpha(x)}=\{t: T(x)(t)=\alpha(x)\}= \begin{cases}\{1\} & \text { if } x \neq 2 \\ \{3\} & \text { if } x=2\end{cases}
$$

and

$$
H(\{1\},\{3\})=\max \left\{\sup _{u \in\{1\}} d(u,\{3\}), \sup _{v \in\{3\}} d(\{1\}, v)\right\}=\frac{5}{17} .
$$

Now,

$$
d_{\infty}(T(3), T(2)) \geq D_{\alpha}(T(3), T(2))=H(\{1\},\{3\})=\frac{5}{17}, \quad d(3,2)=\frac{4}{17} .
$$

Since $d_{\infty}(T(3), T(2))>\alpha d(3,2)$ for each $\alpha<1$ and $X$ is not linear, therefore [12, Theorems $2.1,2.2]$ and main results in $[9-11,16]$ are not applicable to find $1 \in[T 1]_{\frac{3}{4}}$. Now, $[S x]_{\alpha(x)}=\left\{t: S(x)(t)=\frac{3}{4}\right\}=\{1\}$ for all $x \in X$ and

$$
H\left([S x]_{\alpha(x)},[T y]_{\alpha(y)}\right)= \begin{cases}H(\{1\},\{1\})=0 & \text { if } y \neq 2, \\ H(\{1\},\{3\})=\frac{5}{17} & \text { if } y=2 .\end{cases}
$$

It follows that for

$$
a_{1}=a_{2}=a_{3}=a_{5}=0, \quad a_{4}=\frac{5}{17},
$$

$a_{1} d\left(x,[S x]_{\alpha(x)}\right)+a_{2} d\left(y,[T y]_{\alpha(y)}\right)+a_{3} d\left(x,[T y]_{\alpha(y)}\right)+a_{4} d\left(y,[S x]_{\alpha(x)}\right)+a_{5} d(x, y)=\frac{5}{17}$ if $y=2$. Hence, for $a_{1}=a_{2}=a_{3}=a_{5}=0, a_{4}=\frac{5}{17}$, the conditions of Theorem 5 are satisfied to obtain $1 \in[S 1]_{\frac{3}{4}} \cap[T 1]_{\frac{3}{4}}$. Since $a_{3} \neq a_{4}$, the results proved by Vijayaraju and Marudai [17, Theorem 3.1] and Azam and Arshad [15, Theorem 4] are also not applicable. 
Corollary 7 Let $(X, d)$ be a complete metric space and $F, G: X \rightarrow C B(X)$ be multivalued mappings. Suppose that for all $x, y \in X$,

$$
H(F x, G y) \leq a_{1} d(x, F x)+a_{2} d(y, G y)+a_{3} d(x, G y)+a_{4} d(y, F x)+a_{5} d(x, y),
$$

where $a_{1}, a_{2}, a_{3}, a_{4}, a_{5}$ are nonnegative real numbers and $\sum_{i=1}^{5} a_{i}<1$ and either $a_{1}=a_{2}$ or $a_{3}=a_{4}$. Then there exists $u \in X$ such that $u \in F u \cap G u$.

Proof Consider a mapping $\alpha: X \rightarrow(0,1]$ and a pair of fuzzy mappings $S, T: X \rightarrow \mathcal{F}(X)$ defined by

$$
\begin{aligned}
& S(x)(t)= \begin{cases}\alpha(x), & t \in F x, \\
0, & t \notin F x,\end{cases} \\
& T(x)(t)= \begin{cases}\alpha(x), & t \in G x, \\
0, & t \notin G x .\end{cases}
\end{aligned}
$$

Then

$$
[S x]_{\alpha(x)}=\{t: S(x)(t) \geq \alpha(x)\}=F x, \quad[T x]_{\alpha(x)}=G x .
$$

Thus, Theorem 5 can be applied to obtain $u \in X$ such that

$$
u \in[S u]_{\alpha(u)} \cap[T u]_{\alpha(u)}=F u \cap G u .
$$

Corollary 8 [16] Let $(X, d)$ be a complete metric linear space and $S, T: X \rightarrow W(X)$ be fuzzy mappings, and for all $x, y \in X$,

$$
\begin{aligned}
d_{\infty}(S(x), T(y)) \leq & a_{1} p(x, S(x))+a_{2} p(y, T(y))+a_{3} p(x, T(y)) \\
& +a_{4} p(y, S(x))+a_{5} d(x, y)
\end{aligned}
$$

where $a_{1}, a_{2}, a_{3}, a_{4}, a_{5}$ are nonnegative real numbers and $\sum_{i=1}^{5} a_{i}<1$ and either $a_{1}=a_{2}$ or $a_{3}=a_{4}$. Then there exists $u \in X$ such that $\{u\} \subset T(u),\{u\} \subset S(u)$.

Proof Let $x \in X$, then by Lemma 4 there exists $y \in X$ such that $y \in[S x]_{1}$. Similarly, we can find $z \in X$ such that $z \in[T x]_{1}$. It follows that for each $x \in X,[S x]_{\alpha(x)},[T x]_{\alpha(x)}$ are nonempty closed bounded subsets of $X$. As $\alpha(x)=\alpha(y)=1$, by the definition of a $d_{\infty}$-metric for fuzzy sets, we have

$$
H\left([S x]_{\alpha(x)},[T y]_{\alpha(y)}\right) \leq d_{\infty}(S(x), T(y))
$$

for all $x, y \in X$. It implies that

$$
\begin{aligned}
H\left([S x]_{\alpha(x),}[T y]_{\alpha(y)}\right) \leq & d_{\infty}(S(x), T(y)) \\
\leq & a_{1} p(x, S(x))+a_{2} p(y, T(y)) \\
& +a_{3} p(x, T(y))+a_{4} p(y, S(x))+a_{5} d(x, y)
\end{aligned}
$$


for all $x, y \in X$. Since $[S x]_{1} \subseteq[S x]_{\alpha}$ for each $\alpha \in[0,1]$, therefore $d\left(x,[S x]_{\alpha}\right) \leq d\left(x,[S x]_{1}\right)$ for each $\alpha \in[0,1]$. It implies that $p(x, S(x)) \leq d\left(x,[S x]_{1}\right)$, similarly, $p(x, T(x)) \leq d\left(x,[T x]_{1}\right)$. This further implies that for all $x, y \in X$,

$$
\begin{aligned}
H\left([S x]_{1},[T y]_{1}\right) \leq & a_{1} d\left(x,[S x]_{1}\right)+a_{2} d\left(y,[T y]_{1}\right)+a_{3} d\left(x,[T y]_{1}\right) \\
& +a_{4} d\left(y,[S x]_{1}\right)+a_{5} d(x, y) .
\end{aligned}
$$

Now, by Theorem 5 , we obtain $u \in X$ such that $u \in[S u]_{1} \cap[T u]_{1}$, i.e., $\{u\} \subset T(u),\{u\} \subset$ $S(u)$.

In the following, we suppose that $\widehat{T}$ (for details, see $[2,21]$ ) is the set-valued mapping induced by fuzzy mappings $T: X \rightarrow \mathcal{F}(X)$, i.e.,

$$
\widehat{T} x=\left\{y: T(x)(y)=\max _{t \in X} T(x)(t)\right\} .
$$

Corollary 9 Let $(X, d)$ be a complete metric space and $S, T: X \rightarrow \mathcal{F}(X)$ be fuzzy mappings such that for all $x \in X, \widehat{S}(x), \widehat{T}(x)$ nonempty closed bounded subsets of $X$ and

$$
\begin{aligned}
H(\widehat{S} x, \widehat{T} x) \leq & a_{1} d(x, \widehat{S} x)+a_{2} d(y, \widehat{T} y)+a_{3} d(x, \widehat{T} y) \\
& +a_{4} d(y, \widehat{S} x)+a_{5} d(x, y)
\end{aligned}
$$

for all $x, y \in X$, where $a_{1}, a_{2}, a_{3}, a_{4}, a_{5}$ are nonnegative real numbers and $\sum_{i=1}^{5} a_{i}<1$ and either $a_{1}=a_{2}$ or $a_{3}=a_{4}$. Then there exists a point $x^{*} \in X$ such that $T\left(x^{*}\right)\left(x^{*}\right) \geq T\left(x^{*}\right)(x)$ and $S\left(x^{*}\right)\left(x^{*}\right) \geq S\left(x^{*}\right)(x)$ for all $x \in X$.

Proof By Corollary 7, there exists $x^{*} \in X$ such that $x^{*} \in \widehat{S} x^{*} \cap \widehat{T} x^{*}$. Then by [21, Lemma 1$]$, we obtain

$$
T\left(x^{*}\right)\left(x^{*}\right) \geq T\left(x^{*}\right)(x), \quad S\left(x^{*}\right)\left(x^{*}\right) \geq S\left(x^{*}\right)(x)
$$

for all $x \in X$.

Remark 10 The result proved by Vijayaraju and Marudai [17, Theorem 3.1] and Azam and Arshad [15, Theorem 4] is the case when $a_{1} \neq a_{2}$ and $a_{3}=a_{4}$ in Theorem 5. Corollary 9 also generalizes the results proved by Heilpern [9] and Frigon and Regan [12, Theorems 2.1, 2.2] for

$$
\begin{aligned}
& S=T, \\
& a_{i}=0, \quad i=1,2,3,4 \quad \text { and } \quad a_{5} \neq 0 .
\end{aligned}
$$

\section{Conclusion}

In this paper, we obtained fixed point results for fuzzy set-valued mappings under a generalized contractive condition related to the $d_{\infty}$-metric which is useful for computing Hausdorff dimensions. These dimensions help us to understand $e^{\infty}$-space which is used in high energy physics. Our results are also useful in geometric problems arising in high energy physics. This is because events in this case are mostly fuzzy sets. 


\section{Competing interests}

The authors declare that they have no competing interests.

\section{Authors' contributions}

IB gave the idea and AA wrote the initial draft. Both authors read and agreed upon the draft and finalized the manuscript Correspondence was mainly done by IB. All authors read and approved the final manuscript.

\section{Author details}

${ }^{1}$ Department of Mathematics, COMSATS Institute of Information Technology, Islamabad, 44000, Pakistan. ${ }^{2}$ Centre for Applicable Mathematics and Statistics, University of Central Punjab, Lahore, 54770, Pakistan.

\section{Acknowledgements}

Authors are grateful to the editor and referees for their valuable suggestions and critical remarks for improving this paper.

Received: 17 August 2012 Accepted: 9 January 2013 Published: 22 January 2013

\section{References}

1. Weiss, MD: Fixed points and induced fuzzy topologies for fuzzy sets. J. Math. Anal. Appl. 50, 142-150 (1975)

2. Butnariu, D: Fixed point for fuzzy mapping. Fuzzy Sets Syst. 7, 191-207 (1982)

3. Singh, SL, Talwar, R: Fixed points of fuzzy mappings. Soochow J. Math. 19(1), 95-102 (1993)

4. Estruch, VD, Vidal, A: A note on fixed fuzzy points for fuzzy mappings. Proceedings of the II Italian-Spanish Congress on General Topology and Its Applications (Italian) (Trieste, 1999). Rend. Ist. Mat. Univ. Trieste 32(suppl. 2), 39-45 (2001)

5. Wang, G, Wu, C, Wu, C: Fuzzy $\alpha$-almost convex mappings and fuzzy fixed point theorems for fuzzy mappings. Ital J. Pure Appl. Math. 17, 137-150 (2005)

6. Mihet, D: On fuzzy $\epsilon$-contractive mappings in fuzzy metric spaces. Fixed Point Theory Appl. 2007, Article ID 87471 (2007)

7. Qiu, D, Shu, L, Guan, J: Common fixed point theorems for fuzzy mappings under $\varphi$-contraction condition. Chaos Solitons Fractals 41(1), 360-367 (2009)

8. Beg, I, Abbas, M: Invariant approximation for fuzzy nonexpansive mappings. Math. Bohem. 136(1), $51-59$ (2011)

9. Heilpern, S: Fuzzy mappings and fixed point theorems. J. Math. Anal. Appl. 83, 566-569 (1981)

10. Azam, A, Beg, I: Common fixed points of fuzzy maps. Math. Comput. Model. 49, 1331-1336 (2009)

11. Azam, A, Arshad, M, Beg, l: Fixed points of fuzzy contractive and fuzzy locally contractive maps. Chaos Solitons Fractals 42, 2836-2841 (2009)

12. Frigon, M, O'Regan, D: Fuzzy contractive maps and fuzzy fixed points. Fuzzy Sets Syst. 129, $39-45$ (2002)

13. Amemiya, M, Takahashi, W: Fixed point theorems for fuzzy mappings in complete metric spaces. Fuzzy Sets Syst. 125 253-260 (2002)

14. Zhang, W, Qiu, D, Li, Z, Xiong, G: Common fixed point theorems in a new fuzzy metric space. J. Appl. Math. 2012, Article ID 890678 (2012)

15. Azam, A, Arshad, M: A note on 'Fixed point theorems for fuzzy mappings' by P. Vijayaraju and M. Marudai. Fuzzy Sets Syst. 161, 1145-1149(2010)

16. Bose, RK, Sahani, D: Fuzzy mappings and fixed point theorems. Fuzzy Sets Syst. 21, 53-58 (1987)

17. Vijayaraju, P, Marudai, M: Fixed point theorems for fuzzy mappings. Fuzzy Sets Syst. 135, 401-408 (2003)

18. Nadler, SB: Multivalued contraction mappings. Pac. J. Math. 30, 475-488 (1969)

19. Arora, SC, Sharma, V: Fixed points for fuzzy mappings. Fuzzy Sets Syst. 110, 127-130 (2000)

20. Azam, A, Arshad, M, Vetro, P: On a pair of fuzzy $\phi$-contractive mappings. Math. Comput. Model. 52, 207-214 (2010)

21. Shi-sheng, Z: Fixed point theorems for fuzzy mappings (II). Appl. Math. Mech. 7(2), 147-152 (1986)

doi:10.1186/1687-1812-2013-14

Cite this article as: Azam and Beg: Common fuzzy fixed points for fuzzy mappings. Fixed Point Theory and Applications 2013 2013:14

\section{Submit your manuscript to a SpringerOpen ${ }^{\circ}$ journal and benefit from:}

- Convenient online submission

Rigorous peer review

- Immediate publication on acceptance

Open access: articles freely available online

- High visibility within the field

- Retaining the copyright to your article 